\title{
Neutron whispering gallery
}

\author{
Valery V. Nesvizhevsky ${ }^{1 \star}$, Alexei Yu. Voronin ${ }^{2}$, Robert Cubitt ${ }^{1}$ and Konstantin V. Protasov ${ }^{3}$
}

\begin{abstract}
The 'whispering gallery' effect has been known since ancient times for sound waves in air ${ }^{1,2}$, later in water and more recently for a broad range of electromagnetic waves: radio, optics, Roentgen and so on $^{3-6}$. It consists of wave localization near a curved reflecting surface and is expected for waves of various natures, for instance, for atoms ${ }^{7,8}$ and neutrons ${ }^{9}$. For matter waves, it would include a new feature: a massive particle would be settled in quantum states, with parameters depending on its mass. Here, we present for the first time the quantum whispering-gallery effect for cold neutrons. This phenomenon provides an example of an exactly solvable problem analogous to the 'quantum bouncer'10; it is complementary to the recently discovered gravitationally bound quantum states of neutrons ${ }^{11}$. These two phenomena provide a direct demonstration of the weak equivalence principle for a massive particle in a pure quantum state ${ }^{12}$. Deeply bound whispering-gallery states are long-living and weakly sensitive to surface potential; highly excited states are short-living and very sensitive to the wall potential shape. Therefore, they are a promising tool for studying fundamental neutron-matter interactions ${ }^{13-15}$, quantum neutron optics and surface physics effects ${ }^{16-18}$.
\end{abstract}

The classical whispering-gallery phenomenon can be understood in terms of geometrical optics. Thus, neutron Garland trajectories with a high probability of specular reflection have been observed in curved neutron guides ${ }^{19}$ and frequently used in neutron experiments. Elliptical focusing neutron guides using a single reflection are available in commerce. Sound can be reflected in an elliptical chamber from one focus to the other one after 'a single bounce'. A more complex phenomenon consists of wave localization in the vicinity of curved surfaces, also called the whispering-gallery effect. Owing to such localization, the sound can reach a person on the opposite side of a building, or even complete a circle, imitating an 'echo'. Lord Rayleigh explained and described quantitatively this phenomenon in his 'Theory of sound'1,2. He verified the theory using a whistle as a sound source and burning candles as the sound intensity 'detectors'. Whales are believed to communicate over long distances, profiting from a similar effect in surface layers of sea water. The electromagnetic whispering-gallery waves from radio to light ('glory' or 'heiligenschein') and Roentgen frequencies are of ever-growing interest ${ }^{3-6}$ owing to their multiple applications. They are also known as 'Mie scattering' in light scattering from aerosols and in nuclear physics. An optical analogue of a quantum particle bouncing on a hard surface under the influence of gravity or centrifugal potential has been demonstrated recently using a circularly curved optical waveguide ${ }^{20}$. In all of these cases, a curved mirror acts as a waveguide; and interference of the waves falling to the mirror and those reflected causes specific stationary whispering-gallery modes.

For a material wave in a quantum $\operatorname{limit}^{7-9}$, a new feature would appear: radial motion of a massive object would be settled in quantum states, with parameters depending on its mass. At

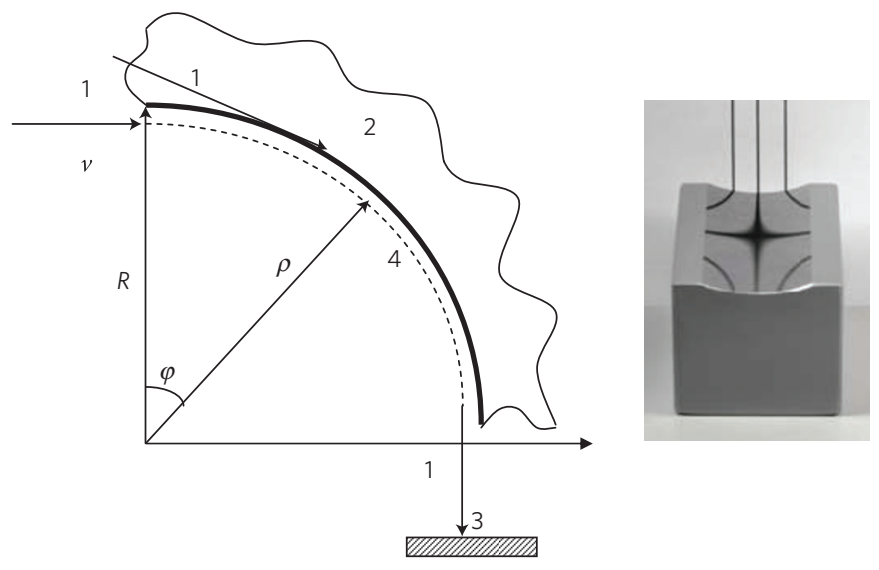

Figure 1 | A scheme of the neutron centrifugal experiment. 1: Classical trajectories of incoming and outgoing neutrons, 2: cylindrical mirror, 3: neutron detector, 4: quantum motion along the mirror surface. Inset: $A$ photo of the single-crystal cylindrical silicon mirror used for the presented experiments, with an optical reflection of black stripes for illustrative purposes.

certain conditions, this problem can be reduced to a quantum particle above a mirror in a linear potential — the so-called quantum bouncer ${ }^{10}$, in analogy to the neutron quantum motion in the Earth's gravitational field above a flat mirror ${ }^{11,21-26}$. Thus, we used similar approaches for the two problems; this analogy motivated the present study to a significant extent, as well as a consideration of a 'neutron centrifuge' in ref. 27.

Consider scattering of a cold neutron with velocity $v \sim 10^{3} \mathrm{~m} \mathrm{~s}^{-1}$ (energy $\varepsilon \sim 10^{-2} \mathrm{eV}$ ) by a perfect cylindrical mirror with a radius $R=2.5 \mathrm{~cm}$ (see Fig. 1$)^{9}$. The mirror is described by a uniform optical potential $U_{0}$ depending on the mirror material (typically $U_{0} \sim 10^{-7} \mathrm{eV}$; ref. 28). The neutron is affected by huge centrifugal acceleration $a_{\text {centr }}=\left(v^{2} / R\right) \sim 10^{5}-10^{7} \mathrm{~g}$ ( $\mathrm{g}$ is the gravitational acceleration on the Earth's surface). In ref. 9, we discussed the limitations of such an interpretation and found that demands on the mirror quality will be extremely high. In particular, the surface roughness should not exceed a few angstroms.

As $\varepsilon \gg U_{0}$, most neutrons entering at a tangential trajectory are deviated to small angles $\varphi$, not larger than twice the classical critical scattering angle $\varphi_{\mathrm{c}}=\sqrt{U_{0} / \varepsilon} \sim 0.2^{\circ}$. However, some neutrons could be captured into long-living centrifugal states and thus could be deviated to large angles. The tangential motion is characterized by the angular momentum quantum number $\mu_{0}=m v R / \hbar$; here $m$ is the neutron mass and $\hbar$ is the reduced Planck constant. Typical values are huge $\mu_{0}=10^{8}-10^{9}$, so quantum effects for tangential motion are negligible. However, for the radial motion, quantum effects are essential. The quantum states are settled in a bounding well formed by the centrifugal potential (approximated

\footnotetext{
${ }^{1}$ Institut Laue-Langevin, 6 rue Jules Horowitz, Grenoble, F-38046, France, ${ }^{2}$ Lebedev Physical Institute, 53 Leninsky prospect, Moscow, 119991, Russia,

${ }^{3}$ Laboratoire de Physique Subatomique et de Cosmologie UJF-CNRS/IN2P3-INPG, Grenoble, F-38026, France. *e-mail: nesvizhevsky@ill.eu.
} 


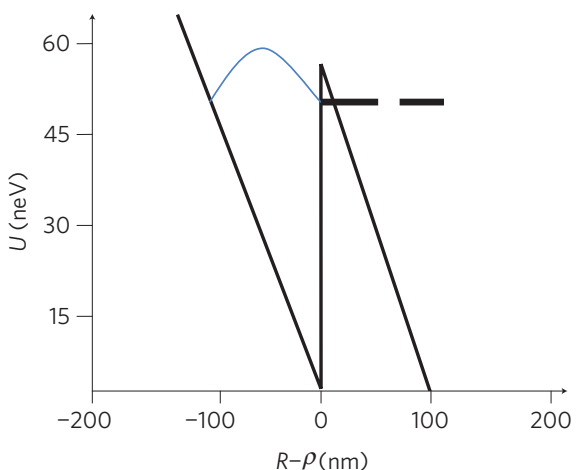

$\lambda_{c}$

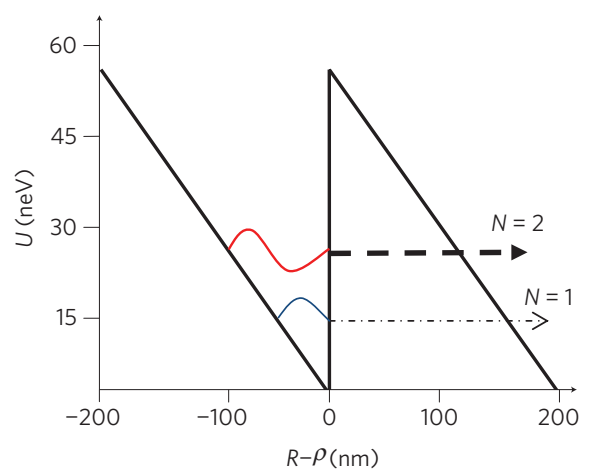

$\lambda>\lambda$

Figure 2 | A sketch of the effective potential in the cylindrical reference system. The potential step at $z=0$ is equal to the mirror optical potential $U_{0}$. The potential slope at $z \neq 0$ is governed by the centrifugal acceleration $a_{\text {centr }}=v^{2} / R$. The wavefunctions of the two lowest quantum states $(n=1,2)$ are shown inside the bounding triangle potential at the height corresponding to their energies. The dashed lines illustrate tunnelling of neutrons through the bounding triangle potential.

by a linear one in the surface vicinity) and the mirror potential shown in Fig. 2. These are quasistationary states, as the probability of tunnelling through the potential barrier is never zero, although it could be negligible for deeply bound states. Similar surface neutron waves have been identified within thin layered structures ${ }^{29}$, with parameters depending on the bounding potential width.

The method to study the quantum states is based on a continuous variation of the triangle barrier width in Fig. 2. The width, the energy and the number of states depend strongly on the velocity $v$. The width decreases exponentially as a function of $v$ owing to the tunnelling through the triangle potential. Small $v$ results in a broad barrier, and this results in a larger number of allowed quantum states and a lower energy of each state. One thus expects a step-like behaviour of the transmitted neutron flux as a function of $v$, using $U_{0}$ as a 'filter' for the quantum states energy. In particular, the transmitted neutron flux increases sharply from zero when the neutron velocity (wavelength) approaches a characteristic cutoff value $v_{c}\left(\lambda_{c}\right)$ corresponding to the appearance of the lowest quasistationary state. It can be estimated from the Heisenberg relation $z_{\mathrm{Cl}} \sqrt{2 m U_{0}}>2 \pi \hbar$; the classical radial motion of a neutron is limited at a height of $z_{\mathrm{Cl}}=\left(R U_{0} / m v^{2}\right)$. Thus, here $\lambda_{\mathrm{c}} \approx 3.9 \AA$, $\lambda(\AA)=3960 / v\left(\mathrm{~m} \mathrm{~s}^{-1}\right)$. The actual cutoff value increases slightly as a function of the deviation angle because of the tunnelling effect.

An alternative method for studying such centrifugal states consists of measuring the radial velocity distribution using a position-sensitive neutron detector, placed at a distance from the mirror. If this distance is large enough, the detection point is unambiguously related to the exit angle. In particular, if a single long-living state is populated, we could measure directly the distribution of radial velocity components in this quantum state. Evidently, the most powerful method consists of combining the two options, that is a simultaneous measurement of $v$ and $\varphi$. The $v$ scan is provided by the standard time-of-flight technique. The angular distributions are measured using a large position-sensitive detector, placed at $3.4 \mathrm{~m}$ distance from the mirror exit. The experiment presented was carried out on $P F 1 B$ and $D 17$ (ref. 30) instruments at the Institut Laue-Langevin; the wavelength range is $2-30 \AA$.

The initial neutron beam width $\left(\sim 10^{-4} \mathrm{~m}\right)$ was much larger than the characteristic quantum state width $\left(\sim 10^{-7} \mathrm{~m}\right)$. The angular divergence was $0.2^{\circ}$. In the first configuration, the neutron beam entered the cylindrical mirror at a tangential trajectory from the mirror bulk; only a small fraction of neutrons tunnelled into the centrifugal quasistationary quantum states. Evidently such neutrons populate only short-living highly excited states. A typical scattering probability as a function of the neutron wavelength and the scattering angle is presented in Fig. 3. In the second configuration, neutrons enter from the entrance edge of a truncated cylindrical mirror; thus, all quantum states could be populated. The fraction of neutrons trapped in the quantum whispering-gallery mode is much larger and the lifetime of deeply bound quantum states is much longer, thus providing much larger deviation angles. A typical result is shown in Fig. 4.

The 'fingerprints' of the quantum states in Figs 3 and 4 have a ' $V$ ' shape. This can be understood as follows. The average deviation angle $\varphi_{0}$ is equal to the angular mirror size; the 'fingerprint' is centred on $\varphi_{0}$. Thus, the radial velocity distribution is symmetric relative to the zero value. For Garland trajectories of neutrons, the width of the ' $\mathrm{V}$ ' shape would be proportional to $\lambda$ for any $\lambda$, as the radial velocity is limited by the mirror critical velocity. The ' $V$ ' shape would point to $\lambda\left(\varphi_{0}\right)=0$, as shown in Figs 3 and 4 with solid lines. An evident manifestation of the observed centrifugal quasistationary states consists of the sharp cutoff in the neutron flux below wavelengths $\lambda_{c}$, corresponding to the appearance of the lowest quantum state, in analogy to the first experiment with the gravitationally bound quantum states of neutrons. Wavelengths in the vicinity of $\lambda_{c}$ are above the maximum intensity in the incoming neutron beam; therefore, the observed cutoff below $\lambda_{c}$ is particularly impressive. The precise values for the cutoffs confirm our theoretical estimation in both cases ${ }^{9}$ with an accuracy of a few per cent that could be largely improved in future. We observe a slight predicted dependence of the cutoffs on the deviation angle $\varphi$, that is on the lifetime, in quantum states. In the classical case no cutoff would exist, as neutrons with any large velocity would pass at sufficiently small distances to the surface.

Another manifestation of the quantum states is the stripe structure inside the ' $\mathrm{V}$ ' shape. It is explained by interference of a few transmitted quantum states. The 'fingerprint' in Fig. 4 has a finer structure owing to the longer times the neutrons have spent in the potential well. The periodic maxima (minima) structure in the observed neutron intensity (stripes) appears at those $\lambda$ and $\varphi$ values that correspond to constructive (destructive) interference of the transmitted quantum states ${ }^{9}$. Figure $4 \mathrm{~b}$ shows a theoretical simulation of the data, which reproduces the measurements in detail. In particular, no interference is observed in the wavelength range below $\sim 5.5 \AA$, as the lowest quantum state alone is populated. Above this wavelength, an interference of the two lowest quantum states appears, producing quasi equal-distance interference lines and so on. The complex structure of the 'fingerprints' is highly sensitive to the shape of the bounding potential in the case of high quantum states and there is no sensitivity for the deeply bound states. This new phenomenon, as well as its many potential applications, has to be explored further. 


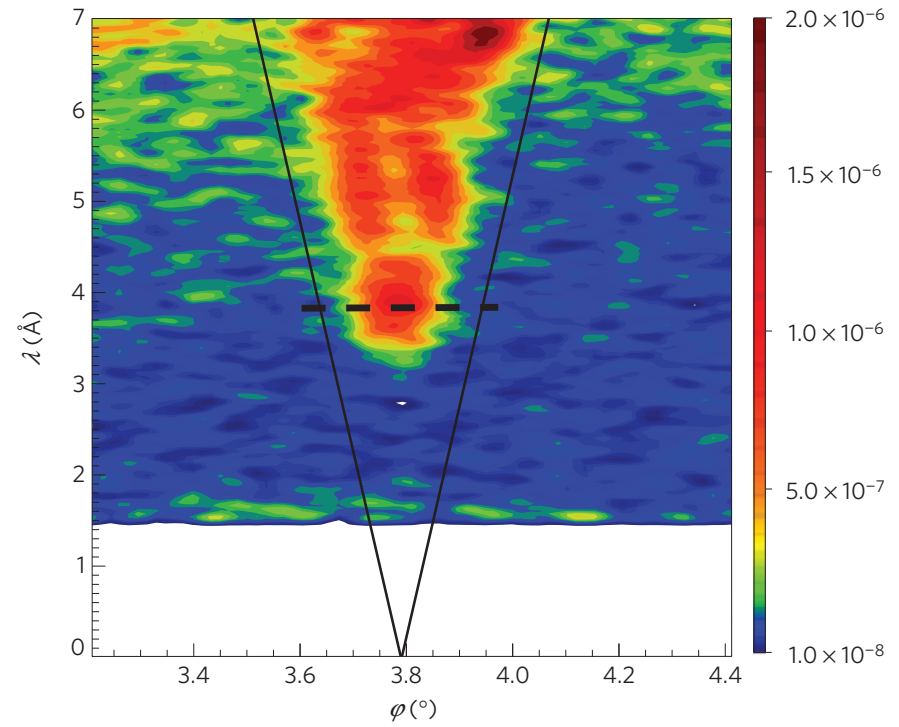

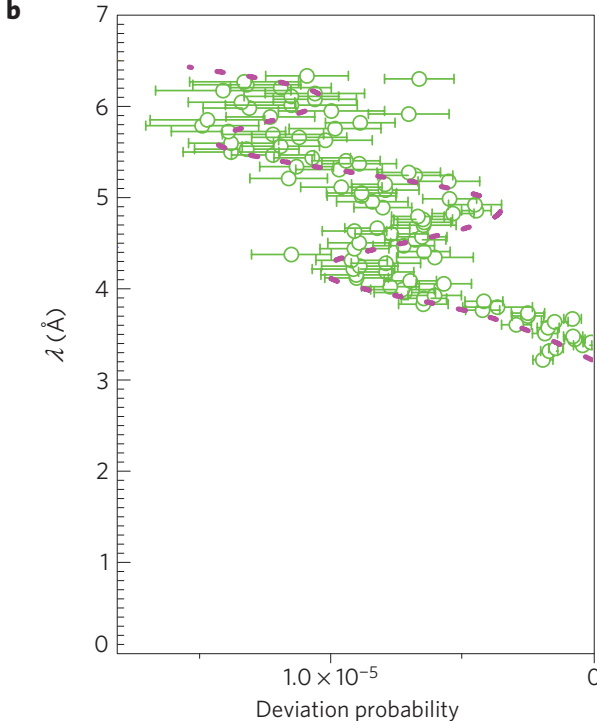

Figure 3 | Short-living centrifugal quantum states. a, The scattering probability as a function of neutron wavelength $\lambda$ ( $\AA$; vertical axis) and deviation angle $\varphi\left(^{\circ}\right.$; horizontal axis). Neutrons enter from the mirror bulk on tangential trajectories. The geometrical exit angle is $3.8^{\circ}$. The inclined solid lines show the signal shape for the classical Garland trajectories. The dashed horizontal line illustrates a characteristic wavelength cutoff $\lambda_{c}$ estimated from the uncertainty principle. $\mathbf{b}$, The deviation probability as a function of the neutron wavelength within the ' $V$ ' shape (projection of the data in $\mathbf{a}$ to the vertical axis): the circles indicate the data points with their standard deviations and the dotted line presents the theoretical expectation.
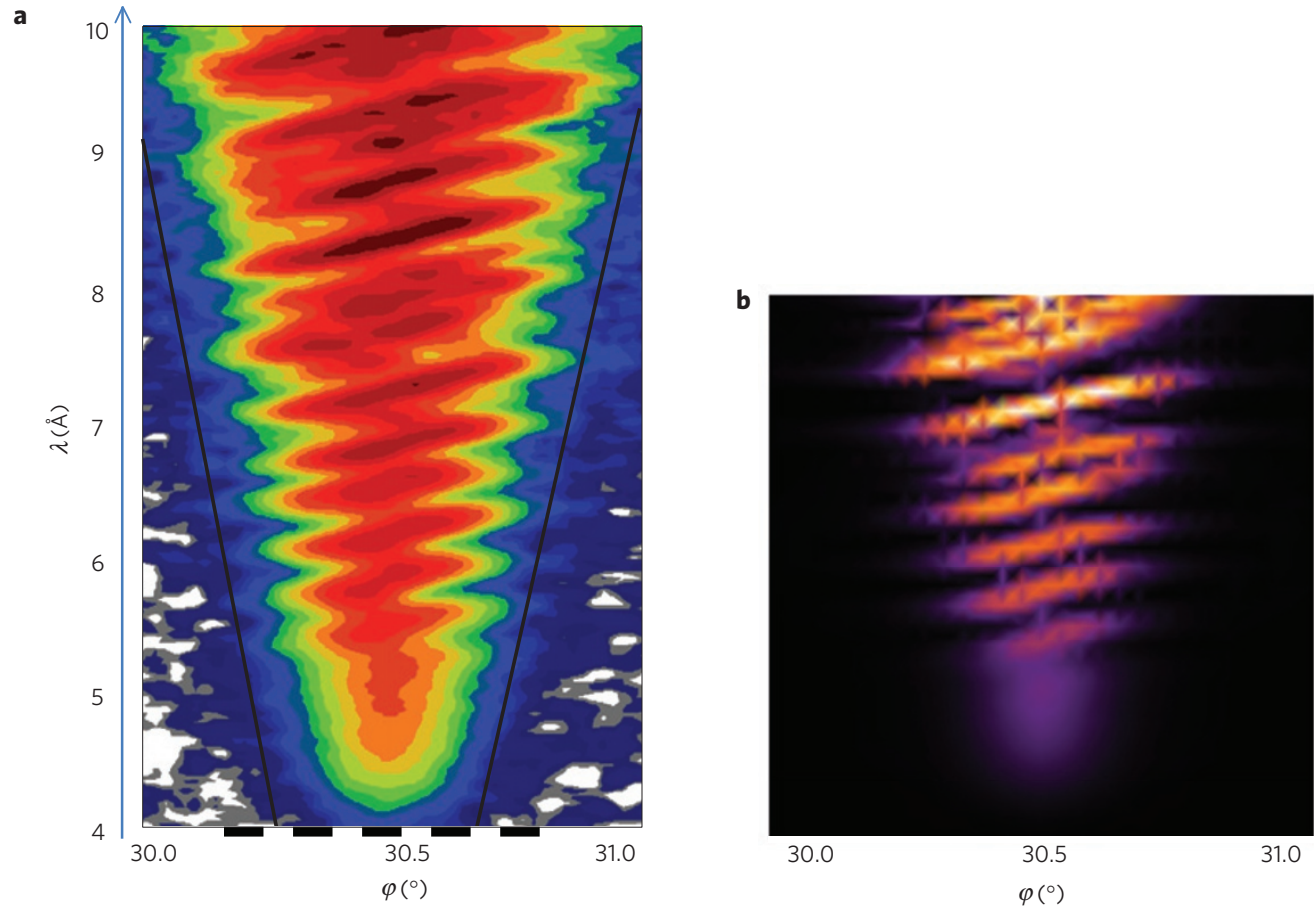

Figure 4 | Long-living centrifugal quantum states. a, The scattering probability as a function of neutron wavelength $\lambda$ ( $\AA$; vertical axis) and deviation angle $\varphi\left({ }^{\circ} ;\right.$ horizontal axis $)$. Neutrons enter through the entrance edge of the mirror. The geometrical angular size of the mirror is $30.5^{\circ}$. The inclined solid lines show the signal shape for the classical Garland trajectories. The dashed horizontal line illustrates a characteristic wavelength cutoff $\lambda_{c}$. $\mathbf{b}$, Theoretical simulation of the data in accordance with refs 9-11. Some of the difference between these two pictures is probably due to the thin oxide layer on the mirror surface.

Combined measurement of the gravitationally bound and centrifugal quantum states of neutrons is a direct demonstration of the weak equivalence principle for a massive body in a pure quantum state ${ }^{12}$; the accuracy is limited by the gravitational experiment ${ }^{23}$ to $10^{-1}$. Although the independence of a free fall on mass does not hold in the quantum limit, quantum states of a massive body in a locally uniform gravitational field and those in a system moving with equal acceleration are equivalent. Both problems, the centrifugal and gravitational ones, provide an excellent experimental laboratory for studying neutron quantum optics phenomena, quantum revivals and localization ${ }^{16-18}$. Evident advantages of using cold neutrons and centrifugal states include the much higher statistics attainable, broad accessibility of cold neutron beams as well as a crucial reduction of many false 
effects owing to $\sim 10^{5}$ times higher energies of the quantum states involved. This phenomenon could provide a promising tool for studying fundamental neutron-matter interactions (in analogy to refs 13-15), as well as surface physics effects. Compared with experiments at standard reflectometers using a single reflection, the centrifugal quantum states have the advantage of at least a few hundred or thousand quasi-classical reflections, providing an extremely high sensitivity to the shape of the bounding potential.

Received 25 June 2009; accepted 3 November 2009; published online 13 December 2009

\section{References}

1. Strutt, J. W. The Theory of Sound Vol. 2 (Macmillan, 1878).

2. Rayleigh, L. Further applications of Bessel's functions of high order to the whispering gallery and allied problems. Phil. Mag. 27, 100-109 (1914).

3. Mie, G. Beitrage zur optic triiben medien speziell kolloidaler metalosungen. Ann. Phys. 25, 377-452 (1908).

4. Debye, P. Der lichtdruck auf kugeln von beleibigem material. Ann. Phys. 30, 57-136 (1909).

5. Oraevsky, A. N. Whispering-gallery waves. Quant. Electron. 32, 377-400 (2002).

6. Vahala, K. J. Optical microcavities. Nature 424, 839-846 (2003).

7. Mabuchi, H. \& Kimble, H. J. Atom galleries for whispering atoms-binding atoms in stable orbits around an optical resonator. Opt. Lett. 19, 749-751 (1994).

8. Vernooy, D. M. \& Kimble, H. J. Quantum structure and dynamics for atom galleries. Phys. Rev. A 55, 1239-1261 (1997).

9. Nesvizhevsky, V. V., Petukhov, A. K., Protasov, K. V. \& Voronin, A. Yu. Centrifugal quantum states of neutrons. Phys. Rev. A 78, 033616 (2008).

10. Flugge, V. Practical Quantum Mechanics Vol. 1 (Springer, 1974).

11. Nesvizhevsky, V. V. et al. Quantum states of neutrons in the Earth's gravitational field. Nature 415, 297-299 (2002).

12. Viola, L. \& Onofrio, R. Testing the equivalence principle through freely falling objects. Phys. Rev. D 55, 455-462 (1997).

13. Bertolami, O. \& Nunes, O. Ultracold neutrons, quantum effects of gravity and the weak equivalence principle. Class. Quant. Gravity 20, L61-L66 (2002).

14. Nesvizhevsky, V. V., Pignol, G. \& Protasov, K. V. Neutron scattering and extra-short-range interactions. Phys. Rev. D 77, 034020 (2008).

15. Baessler, S., Nesvizhevsky, V. V., Protasov, K. V. \& Voronin, A. Yu. Constraint on the coupling of axion-like particles to matter via an ultracold neutron gravitational experiment. Phys. Rev. D 75, 075006 (2007).

16. Robinett, R. Quantum wave packet revivals. Phys. Rep. 392, 1-119 (2004).

17. Mather, W. H. \& Fox, R. F. Coherent-state analysis of the quantum bouncing ball. Phys. Rev. A 73, 032109 (2006).
18. Romera, E. \& Santos, F. de los. Identifying wave-packet fractional revivals by means of information entropy. Phys. Rev. Lett. 99, 263601 (2007).

19. Maier-Leibnitz, H. \& Springer, T. Use of neutron optical devices on beam-hole experiments. J. Nucl. Energy 17, 217-225 (1963).

20. Della Valle, G. et al. Experimental observation of a photon bouncing ball. Phys. Rev. Lett. 102, 180402 (2009)

21. Nesvizhevsky, V. V. et al. Search for quantum states of the neutron in a gravitational field. Nucl. Instrum. Methods 440, 754-759 (2000).

22. Nesvizhevsky, V. V. et al. Measurement of quantum states of neutrons in the Earth's gravitational field. Phys. Rev. D 67, 102002 (2003).

23. Nesvizhevsky, V. V. et al. Study of the neutron quantum states in the gravity field. Eur. Phys. J. C 40, 479-491 (2005).

24. Voronin, A. Yu. et al. Quantum motion of a neutron in a waveguide in the gravitational field. Phys. Rev. D 73, 044029 (2006).

25. Adhikari, R., Cheng, Y., Meyerovich, A. E. \& Nesvizhevsky, V. V. Quantum size effect and biased diffusion of gravitationally bound neutrons in a rough waveguide. Phys. Rev. A 75, 063613 (2007).

26. Westphal, A. et al. A quantum mechanical description of the experiment on the observation of gravitationally bound states. Eur. Phys. J. C 51, 367-375 (2007).

27. Watson, P. Bouncing neutron and the neutron centrifuge. J. Phys. G 29, 1451-1462 (2003)

28. Fermi, E. A Course in Neutron Physics (Collected Papers, Univ. Chicago Press, 1965).

29. Pfeiffer, F. et al. Submicrometre coherent beam production using a thin-film waveguide. Phys. Rev. Lett. 88, 055507 (2002).

30. Cubitt, R. \& Fragneto, G. D17: The new reflectometer at the ILL. Appl. Phys. A 74, S329-S344 (2002).

\section{Acknowledgements}

We are grateful to our colleagues from the GRANIT collaboration and students, in particular H. G. Börner, C. Codau, Ch. Dornes, P. A. Gurzhiyants, A. K. Petukhov, G. Pignol, T. Soldner and A. V. Strelkov, for stimulating discussions and help, and also to ANR (Agence Nationale de la Recherche, France) for support of this work in the framework of ANR-05-BLAN-0098-01. The mirror was produced by SESO.

\section{Author contributions}

V.V.N. proposed the concept and carried out preliminary studies; R.C. and V.V.N. carried out the measurement and analysed the data; A.Yu.V. developed the theory; K.V.P. and V.V.N. contributed to the theory; all authors contributed to writing the manuscript.

\section{Additional information}

The authors declare no competing financial interests. Reprints and permissions information is available online at http://npg.nature.com/reprintsandpermissions. Correspondence and requests for materials should be addressed to V.V.N. 